\title{
Comportamiento de aceites poliinsaturados en la preparación de patatas fritas para consumo inmediato: Formación de nuevos compuestos y comparación de métodos analíticos
}

\author{
Por L. Masson'1, P. Robert1, N. Romero', M. Izaurieta', S. Valenzuela', J. Ortiz'1 y M. C. Dobarganes \\ 1. Departamento de Ciencia de los Alimentos y Tecnología Química, Facultad de Ciencias Químicas y \\ Farmacéuticas, Universidad de Chile. Casilla 233, Santiago 1, Chile. \\ 2. Instituto de la Grasa (CSIC). Avda. Padre García Tejero, 4. 41012 - Sevilla.
}

\begin{abstract}
RESUMEN
Comportamiento de aceites poliinsaturados en la preparación de patatas fritas para consumo inmediato: Formación de nuevos compuestos y comparación de métodos analíticos.

En este estudio se aplican diferentes métodos analíticos -compuestos polares, distribución de compuestos polares, acidez, índice de peróxidos, dienos y trienos conjugados, ácidos oxidados insolubles en éter de petróleo, viscosidad y ensayo oxifrit- a muestras de aceites de soja, girasol y mezclas de soja y colza, obtenidas en establecimientos públicos, antes y después de ser usadas en la fritura de patatas. Los dos objetivos principales fueron: conocer los compuestos formados durante el proceso de fritura de patatas y evaluar la posibilidad de sustitución de determinaciones complejas por métodos analíticos más simples y rápidos.

Los resultados indicaron que, aunque los compuestos de polimerización y oxidación son los más representativos entre los nuevos compuestos originados durante el proceso, un $20 \%$ de las muestras contenían cantidades anormalmente elevadas de compuestos de hidrólisis cuya presencia es difícil de explicar. A partir de las correlaciones existentes entre métodos analíticos se puede deducir que, en este sector, la acidez libre y el porcentaje de dienos conjugados (o viscosidad), son útiles para predecir las cantidades de los principales grupos de nuevos compuestos presentes en los aceites de fritura poliinsaturados.
\end{abstract}

PALABRAS-CLAVE: Aceite de fritura (calidad) - Aceite poliinsaturado - Compuestos polares - Método analítico rápido Patatas.

\section{SUMMARY}

Performance of polyunsaturated oils during frying of potatoes in fast food shops: Formation of new compounds and correlations between analytical methods.

In this study, different analytical methods, i.e. polar compounds, polar compound distribution, free fatty acids, peroxide value, conjugated dienes and trienes, oxidized fatty acids, viscosity and oxifrit-test, are applied to samples of both initial polyunsaturated oils and their counterparts after being used in the frying of potatoes in fast food shops. Two main objectives were established: the study of formation of new compounds during the frying process and the evaluation of possibilities to substitute complex methodologies for simpler and more rapid techniques.

Results indicated that, although new compounds comming from oxidation and polymerization stand out, $20 \%$ of the samples contained very high amounts of hydrolytic products i.e. diglycerides and fatty acids, whose formation is difficult to explain.
From the analysis of correlation coefficients between analytical methods, the utility of free fatty acids and conjugated dienes (or viscosity) to predict the amount of the main groups of new compounds in used polyunsaturated frying oils, was deduced.

KEY-WORDS: Frying oil (quality) - Polar compounds Polyunsaturated oil - Potatoes - Rapid analytical method.

\section{INTRODUCCIÓN}

La creciente utilización de grasas y aceites comestibles para la preparación de productos fritos, obliga cada vez más a un estricto control de los aceites y grasas de fritura para mantener la calidad de los alimentos fritos y garantizar su seguridad. Con este objetivo, se han establecido recomendaciones o normas de obligado cumplimiento en diferentes países que tratan de limitar la utilización de los aceites y grasas de fritura para consumo humano (Firestone, 1996).

El método analítico de aplicación más general para la evaluación de la calidad de las grasas de fritura es la determinación de compuestos polares mediante cromatografía de adsorción en columna de sílice, que proporciona una medida exacta y reproducible del porcentaje de compuestos presentes en la grasa con mayor polaridad que los triglicéridos, la mayor parte de los cuales son compuestos de degradación originados durante el proceso de fritura (IUPAC, 1987). Esta determinación es también la más utilizada en las normativas existentes para limitar la alteración de los aceites y grasas de fritura, que se ha establecido en torno al $25 \%$.

Aunque en el momento actual el análisis de los aceites y grasas de fritura no presenta especiales problemas, la atomización del sector y la carencia de facilidades de laboratorio en los establecimientos de preparación de alimentos fritos, limitan el cumplimiento de las normas de calidad existentes y exigen de las Administraciones Públicas la selección y propuesta de métodos alternativos simples que puedan ser utilizados por el usuario de la freidora (Dobarganes et al., 1989). 
Existe una amplia variedad de métodos analíticos que son utilizados para la evaluación de las grasas y aceites de fritura (Stevenson, 1984; White, 1991; Orthoefer y Cooper, 1996). Por una parte, se dispone de métodos que cuantifican de forma directa los compuestos de alteración originados, entre los cuales destacan la determinación de compuestos polares citada y la determinación específica de los compuestos de polimerización (Wolf, 1992), oxidación e hidrólisis (Dobarganes, 1988), relacionados con los tres tipos de alteraciones más importantes que se dan en el proceso de fritura.

Frente a este grupo de métodos, se encuentran los índices analíticos rápidos, sencillos y reproducibles de poca utilidad para la evaluación de aceites de historia desconocida, pero que pueden ser de gran interés para conocer la evolución de la grasa de fritura en aplicaciones concretas. Entre ellos se encuentran la acidez libre, los métodos colorimétricos basados en indicadores redox, o las medidas de viscosidad y dienos conjugados, entre otros.

La propuesta de métodos analíticos simples que sean de ayuda en la mejora de la calidad de los aceites y grasas de fritura, es un objetivo que debe ser alcanzado a medio-largo plazo. La sustitución de la determinación establecida en las normas que limitan su utilización, debe estar basada en la demostración de que existe una buena correlación entre los compuestos polares y el método más simple seleccionado. Sin embargo, los resultados de estudios previos que establecen correlaciones entre métodos analíticos, son poco útiles para establecer conclusiones de carácter general. Así, por ejemplo, se han encontrado coeficientes de correlación entre la cantidad de compuestos polares y la acidez libre tan variables como 0.28 (Al-Kathani, 1991), 0.33 (Croon et al., 1986), 0.37 (Pérez-Camino et al., 1988) y 0.92 (Smith et al., 1986). La variabilidad de los resultados se debe, sin duda, a que no existe una buena correlación general entre ambos métodos, lo que no impide que puedan obtenerse valores elevados dependiendo de las características de las muestras. Este hecho se demuestra fácilmente cuando se conoce el origen de los aceites de fritura y se separan en grupos de mayor homogeneidad. Partiendo de un coeficiente de correlación bajo y sin significación estadística, es posible encontrar coeficientes elevados para distintos grupos de muestras en las que se dan valores más restringidos de las variables del proceso de fritura (Pérez-Camino et al, 1988).

Del mismo modo, para aquellos métodos simples cuyos valores dependen del grado de insaturación del aceite, como es el caso de la viscosidad, los coeficientes de correlación pueden ser elevados sólo cuando se consideran por separado los aceites y grasas con similar insaturación (Suys, 1991).

Teniendo en cuenta estos antecedentes, en este estudio se limita el aceite de fritura a los aceites poliinsaturados. Además, se utiliza la patata, que constituye con gran diferencia el producto frito de mayor consumo, como sustrato exclusivo del proceso, con dos objetivos fundamentales:

a) Analizar la formación de nuevos compuestos en aceites de fritura utilizados en la preparación de un único producto frito.

b) Analizar las correlaciones existentes entre las cantidades de compuestos de alteración y métodos analíticos más simples para deducir si la homogeneidad en el aceite y producto permite sugerir un método aplicable en sustitución de la determinación oficial.

\section{PARTE EXPERIMENTAL}

\section{Muestras y tratamientos}

Se han analizado dos series de quince muestras de aceites poliinsaturados -ocho aceites de soja, cinco aceites de girasol y dos mezclas de aceites de soja y colza de bajo contenido en ácido erúcico- recogidas en quince establecimientos de Santiago (Chile) dedicados a la preparación de patatas fritas tipo bastón. Las muestras corresponden a los aceites frescos (serie A) y después de haber sido utilizados en la fritura de patatas fritas (serie B).

Las muestras utilizadas en fritura fueron tomadas de las correspondientes freidoras discontinuas $y$, con excepción del tipo de aceite utilizado y el producto frito, pueden diferir en el resto de las variables del proceso: tiempo de fritura, días de uso, cantidad de aceite de reposición utilizado, temperatura, diseño de la freidora, etc. Las muestras se mantienen congeladas a $-20^{\circ} \mathrm{C}$ hasta el momento del análisis.

\section{Determinaciones analíticas}

Determinación de compuestos polares.- Se utiliza la metodología propuesta por la IUPAC en la Norma 2.507 (IUPAC, 1987).

Distribución de compuestos polares. - La determinación se realiza mediante cromatografía de exclusión, partiendo de una solución de 10-15 mg/mL de compuestos polares en tetrahidrofurano. La separación se efectúa mediante dos columnas en serie de 100 y 500 A utilizándose como fase móvil tetrahidrofurano, con un flujo de $1 \mathrm{~mL} / \mathrm{min}$. El análisis permite la determinación cuantitativa de los polímeros de triglicéridos, dímeros de triglicéridos, monómeros de triglicéridos oxidados, diglicéridos y ácidos grasos (Dobarganes et al., 1988).

Acidez libre.- Se sigue la metodología propuesta por la AOCS en la Norma Ac5-41 (AOCS, 1993).

Índice de peróxidos.- Se sigue la metodología propuesta por la AOCS en la Norma Cd8-53 (AOCS, 1993). 
Dienos conjugados.- Se sigue la metodología propuesta por la AOCS en la Norma Ti1a-64 (AOCS, 1993).

Trienos conjugados. - Se sigue la metodología propuesta por la AOCS en la Norma Cd7-58 (AOCS, 1993).

Determinación de ácidos oxidados insolubles en éter de petróleo. - Se sigue la metodología establecida por la IUPAC en la Norma 2.502 (IUPAC, 1987).

Viscosidad.-Se realiza la determinación a $40 \pm 1^{\circ} \mathrm{C}$ utilizando el viscosímetro Brookfield, Modelo LVT con adaptador de ultra baja viscosidad a 6 r.p.m.

Prueba colorimétrica Oxifrit.- Se realiza el ensayo en las condiciones establecidas por la firma que comercializa la prueba (Mayer, 1979).

\section{RESULTADOS Y DISCUSIÓN}

La tabla I resume las características generales de los aceites iniciales obtenidos en los quince establecimientos seleccionados. En general, puede observarse que los valores de los índices más representativos de la calidad -acidez libre, índice de peróxidos, dienos y trienos conjugados- son indicativos de aceites bien refinados. Las restantes determinaciones -compuestos polares, ácidos oxidados insolubles en éter de petroleo y viscosidad- se encuentran entre las más utilizadas para la evaluación de las grasas de fritura y sus valores iniciales son de interés para conocer la posterior evolución de los aceites una vez sometidos al proceso. Los porcentajes de compuestos polares son, en general, inferiores al $5 \%$, lo que indica muy buenas características como aceites de fritura y los valores de viscosidad son muy homogéneos debido al similar grado de insaturación de los aceites. Los resultados más sorprendentes y heterogéneos corresponden a la determinación de ácidos oxidados insolubles en éter de petróleo que, incluso en algún caso, sobrepasan el valor del $1 \%$ establecido en las recomendaciones iniciales de la Sociedad Alemana de Investigación en Grasas para desechar los aceites y grasas de fritura (Guhr y Waibel, 1979).

Las tablas II y III recogen los resultados obtenidos para los aceites ya usados en fritura. En la tabla II se detalla la evaluación de los principales grupos de compuestos de degradación originados durante el proceso de fritura. En la primera columna se encuentran los compuestos polares totales y, en las tres siguientes, se resume su distribución en distintos grupos de compuestos obtenidos mediante cromatografía de exclusión. Aunque como se indica en la parte experimental, la técnica permite la separación de cinco grupos de compuestos de peso molecular diferenciado, los resultados se han simplificado en tres grupos de distinta significación, los compuestos de polimerización característicos de la alteración térmica, los triglicéridos oxidados originados exclusivamente por la acción del oxígeno del aire, y los compuestos de hidrólisis atribuidos a la acción de la humedad.

Tabla I

Características generales de los aceites iniciales

\begin{tabular}{|c|c|c|c|c|c|c|c|c|}
\hline Muestra & Aceite & $\begin{array}{c}\text { Acidez } \\
\text { (\%oleico) }\end{array}$ & $\begin{array}{l}\text { Índice de } \\
\text { Peróxidos } \\
(\mathrm{mq} / \mathrm{Kg})\end{array}$ & $\begin{array}{c}\text { Dienos } \\
\text { Conjugados } \\
(\%)\end{array}$ & $\begin{array}{c}\text { Trienos } \\
\text { Conjugados } \\
(\%)\end{array}$ & $\begin{array}{c}\text { Compuestos } \\
\text { Polares } \\
(\%)\end{array}$ & $\begin{array}{c}\text { Ácidos } \\
\text { Oxidados } \\
(\%)\end{array}$ & $\begin{array}{l}\text { Viscosidad } \\
\text { (CP) }\end{array}$ \\
\hline $1 \mathrm{~A}$ & Soja/Colza & 0,05 & 3,3 & 0,6 & 0,04 & 5.4 & 0,8 & 33,1 \\
\hline $2 A$ & Girasol & 0,05 & 3,9 & 0,4 & 0,08 & 4,4 & 0,4 & 32,5 \\
\hline $3 A$ & Soja/Colza & 0,06 & 5,2 & 0,4 & 0,02 & 4,8 & 0,7 & 33,5 \\
\hline $4 \mathrm{~A}$ & Soja & 0,06 & 4,5 & 0,4 & 0,05 & 4,2 & 0,4 & 30,5 \\
\hline $5 A$ & Girasol & 0,05 & 1,6 & 0,4 & 0,08 & 5,1 & 0,4 & 33,5 \\
\hline $6 \mathrm{~A}$ & Soja & 0,07 & 3,1 & 0,5 & 0,05 & 3,1 & 0,5 & 33,0 \\
\hline $7 A$ & Soja & 0,10 & 2,5 & 0,7 & 0,00 & 3,7 & 1,3 & 33,0 \\
\hline $8 \mathrm{~A}$ & Soja & 0,10 & 2,5 & 0,4 & 0,03 & 4,7 & 0,1 & 34,0 \\
\hline $9 A$ & Girasol & 0,08 & 2,5 & 0,3 & 0,06 & 3,7 & 0,2 & 31,5 \\
\hline $10 \mathrm{~A}$ & Soja & 0,50 & 5,3 & 0,4 & 0,03 & 2,4 & 0,1 & 31,0 \\
\hline $11 \mathrm{~A}$ & Girasol & 0,06 & 2,5 & 0,6 & 0,07 & 4,9 & 0,8 & 31,0 \\
\hline $12 \mathrm{~A}$ & Soja & 0,12 & 2,5 & 0,8 & 0,00 & 4,6 & 0,4 & 34,5 \\
\hline $13 A$ & Soja & 0,09 & 2,5 & 0,5 & 0,00 & 4,2 & 0,7 & 32,0 \\
\hline $14 \mathrm{~A}$ & Girasol & 0,09 & 2,5 & 0,6 & 0,02 & 9,8 & 0,2 & 31,2 \\
\hline $15 A$ & Soja & 0,08 & 2,5 & 0,6 & 0,06 & 3,3 & 0,1 & 31,2 \\
\hline
\end{tabular}


Tabla II

Compuestos polares (\%), distribución de compuestos polares (\%) en muestras utilizadas en la fritura de patatas

\begin{tabular}{|c|c|c|c|c|}
\hline \multirow[b]{2}{*}{ Muestra } & \multirow[b]{2}{*}{$\begin{array}{l}\text { Compuestos } \\
\text { Polares (\%) }\end{array}$} & \multicolumn{3}{|c|}{ DISTRIBUCIÓN } \\
\hline & & Polímerosa & $\begin{array}{c}\text { Monómeros } \\
\text { oxidados }\end{array}$ & $\begin{array}{l}\text { b Compuestos } \\
\text { de hidrólisis }\end{array}$ \\
\hline $1 \mathrm{~B}$ & 12,1 & $\begin{array}{c}6,0 \\
(49,6)^{\star}\end{array}$ & $\begin{array}{c}4,9 \\
(40,5)\end{array}$ & $\begin{array}{c}1,2 \\
(9,9)\end{array}$ \\
\hline $2 B$ & 8,8 & $\begin{array}{c}3,0 \\
(34,2)\end{array}$ & $\begin{array}{c}2,9 \\
(32,9)\end{array}$ & $\begin{array}{c}2,9 \\
(32,9)\end{array}$ \\
\hline $3 B$ & 16,7 & $\begin{array}{c}8,3 \\
(49,7)\end{array}$ & $\begin{array}{c}6,7 \\
(40,1)\end{array}$ & $\begin{array}{c}1,7 \\
(10,2) .\end{array}$ \\
\hline $4 \mathrm{~B}$ & 4,3 & $\begin{array}{c}0,7 \\
(12,6)\end{array}$ & $\begin{array}{c}1,8 \\
(41,9)\end{array}$ & $\begin{array}{c}1,8 \\
(41,9)\end{array}$ \\
\hline $5 B$ & 12,5 & $\begin{array}{c}5,5 \\
(44,0)\end{array}$ & $\begin{array}{c}3,0 \\
(24,0)\end{array}$ & $\begin{array}{c}4,0 \\
(32,0)\end{array}$ \\
\hline $6 B$ & 18,6 & $\begin{array}{c}3,9 \\
(21,0)\end{array}$ & $\begin{array}{c}2,1 \\
(11,3)\end{array}$ & $\begin{array}{c}12,6 \\
(67,7)\end{array}$ \\
\hline $7 B$ & 18,9 & $\begin{array}{c}11,4 \\
(60,3)\end{array}$ & $\begin{array}{c}5,5 \\
(29,1)\end{array}$ & $\begin{array}{c}2,0 \\
(10,6)\end{array}$ \\
\hline $8 B$ & 25,4 & $\begin{array}{c}14,3 \\
(56,3)\end{array}$ & $\begin{array}{c}9,7 \\
(38,2)\end{array}$ & $\begin{array}{c}1,4 \\
(5,5)\end{array}$ \\
\hline $9 \mathrm{~B}$ & 21,3 & $\begin{array}{c}2,7 \\
(12,6)\end{array}$ & $\begin{array}{c}1,3 \\
(6,1)\end{array}$ & $\begin{array}{c}17,3 \\
(81,2)\end{array}$ \\
\hline $10 \mathrm{~B}$ & 13,7 & $\begin{array}{c}6,4 \\
(46,7)\end{array}$ & $\begin{array}{c}5,2 \\
(40,0)\end{array}$ & $\begin{array}{c}2,1 \\
(15,2)\end{array}$ \\
\hline $11 \mathrm{~B}$ & 5,3 & $\begin{array}{c}1,7 \\
(32,0)\end{array}$ & $\begin{array}{c}1,8 \\
(34,0)\end{array}$ & $\begin{array}{c}1,8 \\
(34,0)\end{array}$ \\
\hline $12 \mathrm{~B}$ & 10,8 & $\begin{array}{c}5,7 \\
(52,8)\end{array}$ & $\begin{array}{c}3,8 \\
(35,2)\end{array}$ & $\begin{array}{c}1,3 \\
(12,0)\end{array}$ \\
\hline $13 B$ & 5,3 & $\begin{array}{c}2,1 \\
(39,6)\end{array}$ & $\begin{array}{c}2,3 \\
(43,4)\end{array}$ & $\begin{array}{c}0,9 \\
(17,0)\end{array}$ \\
\hline $14 \mathrm{~B}$ & 16,5 & $\begin{array}{c}9,8 \\
(59,4)\end{array}$ & $\begin{array}{c}4,7 \\
(28,5)\end{array}$ & $\begin{array}{c}2,0 \\
(12,1)\end{array}$ \\
\hline $15 \mathrm{~B}$ & 15,2 & $\begin{array}{c}5,2 \\
(34,2)\end{array}$ & $\begin{array}{c}2,4 \\
(15,8)\end{array}$ & $\begin{array}{c}7,6 \\
(50,0)\end{array}$ \\
\hline
\end{tabular}

* \% sobre los compuestos polares totales.

apolímeros y dímeros de triglicéridos; bTriglicéridos oxidados monómeros; cDiglicéridos y Acidos grasos

Como puede observarse, las cantidades encontradas en las distintas muestras son muy variables por ser aceites muestreados al azar que, por tanto, se diferencian en muchas de las variables del proceso y, específicamente, en el tiempo de utilización. Por ejemplo, las muestras 4,11 y 13 tienen contenidos en compuestos polares muy similares a los aceites iniciales lo que indicaría muy poco tiempo de fritura mientras las muestras $6,7,8$ y 9 tienen ya una degradación apreciable. Es interesante destacar que sólo la muestra 8 tiene un porcentaje de compuestos polares en torno al
$25 \%$ y, por ello, debería ser desechada de acuerdo con las recomendaciones existentes.

Es interesante resaltar el bajo nivel medio de alteración de las muestras en contraste con los resultados de estudios previos donde se evalúan muestras de aceites y grasas de fritura obtenidas por los Servicios de Inspección en el sector de restauración de distintos países (Croon et al., 1986; Gertz, 1986; Sebedio et al., 1987; Dobarganes y Márquez-Ruiz, 1995; Skrokki, 1995). En todos ellos se encuentra que más de un $30 \%$ de las muestras analizadas contenían cantidades de compuestos polares por encima del $25 \%$ y no cumplían, en consecuencia, con las normas de calidad establecidas. La diferencia fundamental con los estudios previos radica en que, en estos estudios, la patata es utilizada como único sustrato del proceso de fritura. Es posible que la cantidad de aceite que incorpora la patata durante la fritura sea superior a la de otros productos fritos y ello origine una mayor velocidad de reposición con aceite fresco que, a su vez, contribuye a disminuir la degradación del aceite.

Los resultados más sorprendentes en este estudio corresponden a la distribución de los compuestos polares encontrada en las muestras de fritura. En principio, cabría esperar que aceites de similar grado de insaturación, utilizados en la elaboración de un mismo producto cuyo contenido lipídico es menor del $1 \%$, tuvieran un comportamiento parecido dada la importancia de las dos variables comunes. Ello implicaría que aceites con niveles de compuestos polares del mismo orden deberían tener una distribución similar. Los resultados, sin embargo, indican variabilidades extremas de gran interés. Así, por ejemplo, las muestras 6,9 y 15 destacan por su contenido excepcionalmente elevado en compuetos de hidrólisis normalmente atribuidos a la acción de la humedad del alimento que, sin embargo, es del mismo orden en la patata, independientemente de la variedad.

Específicamente, las muestras 6 y 7 , que tienen igual cantidad de compuestos polares difieren de forma extrema en la distribución de los mismos. Mientras un $67,7 \%$ de los compuestos polares en la muestra 6 corresponden a diglicéridos y ácidos grasos, la muestra 7 sólo contiene algo más de un $10 \%$.

Los resultados obtenidos son de interés por dos razones:

1. Por una parte, estudios previos de fritura, realizadas en laboratorio en condiciones bien controladas, utilizando aceites y grasas de muy distinto grado de insaturación, demuestran que la alteración hidrolítica es la de menor importancia cuantitativa a pesar del elevado grado de humedad de la patata (Sebedio et al., 1990; Pérez-Camino, et al., 1992; Arroyo et al., 1992; Arroyo et al., 1993; Dobarganes et al., 1993; Cuesta et al., 1995). Los resultados encontrados en un $20 \%$ de las muestras reales analizadas son suficientemente significativos para inducir a pensar en la existencia de una variable no controlada que origina la hidrólisis del aceite. Un análisis detallado de los estu- 
Tabla III

Características fisicoquímicas de los aceites usados en fritura

\begin{tabular}{cccccccc}
\hline Muestra & $\begin{array}{c}\text { Acidez } \\
(\% \text { oleico) }\end{array}$ & $\begin{array}{c}\text { Índice de } \\
\text { Peróxidos } \\
(\mathrm{mq} / \mathrm{Kg})\end{array}$ & $\begin{array}{c}\text { Dienos } \\
\text { Conjugados } \\
(\%)\end{array}$ & $\begin{array}{c}\text { Trienos } \\
\text { Conjugados } \\
(\%)\end{array}$ & $\begin{array}{c}\text { Ácidos } \\
\text { Oxidados } \\
(\%)\end{array}$ & $\begin{array}{c}\text { Viscosidad } \\
(\mathrm{CP})\end{array}$ & Oxifrit \\
\hline 1B & 0,16 & 6,4 & 1,3 & 0,03 & 1,2 & 41,0 & $-^{*}$ \\
2B & 0,38 & 4,7 & 0,8 & 0,08 & 0,4 & 33,0 & - \\
3B & 0,31 & 11,9 & 1,9 & 0,04 & 1,0 & 42,5 & - \\
4B & 0,07 & 5,4 & 0,5 & 0,05 & 0,5 & 30,8 & - \\
5B & 0,88 & 2,9 & 1,2 & 0,07 & 0,5 & 35,4 & - \\
6B & 2,62 & 4,0 & 1,1 & 0,09 & 0,7 & 34,0 & - \\
7B & 0,40 & 5,2 & 2,6 & 0,02 & 1,5 & 44,1 & - \\
8B & 0,24 & 5,0 & 2,4 & 0,11 & 1,9 & 47,4 & $+^{\star *}$ \\
9B & 4,80 & 5,3 & 1,0 & 0,09 & 1,1 & 34,0 & - \\
10B & 1,67 & 8,2 & 1,5 & 0,05 & 1,1 & 34,0 & - \\
11B & 0,11 & 2,5 & 0,6 & 0,07 & 0,4 & 33,0 & - \\
12B & 0,18 & 4,9 & 1,4 & 0,04 & 0,6 & 36,5 & - \\
13B & 0,14 & 2,5 & 1,2 & 0,02 & 0,7 & 32,5 & - \\
14B & 0,34 & 5,1 & 0,8 & 0,06 & 2,0 & 32,5 & - \\
15B & 1,83 & 8,3 & 1,7 & 0,06 & 1,1 & 35,8 & - \\
\hline
\end{tabular}

* El aceite puede seguir en uso

** El aceite debe ser desechado

dios previos muestra que la temperatura, establecida alrededor de $180^{\circ} \mathrm{C}$, es la única variable similar en todas las experiencias, existiendo grandes diferencias en el resto de las variables de importancia en el proceso: tipo de aceite, número de operaciones de fritura, tiempo de calentamiento, velocidad de reposición con aceite fresco, y relación superficie/volumen de aceite. Podría especularse, por tanto, que la formación de compuestos de hidrólisis está relacionada con la aplicación de una temperatura muy inferior a la usual del proceso o a la existencia de algún tratamiento dado a la patata antes de la fritura en determinados establecimientos.

2. Por otra parte, la diferente distribución de compuestos polares aumenta el interés de la determinación mediante cromatografía de exclusión, dada la distinta significación de los compuestos de termoxidación e hidrólisis. Si el límite establecido tiene como objetivo el garantizar la seguridad de los alimentos fritos, es indudable que la ingestión de aceites con similares cantidades de compuestos polares tendría diferente significación nutricional dependiendo de las cantidades relativas de compuestos de termoxidación e hidrólisis, ya que mientras los primeros contienen ácidos grasos alterados sospechosos de modificar negativamente el valor nutricional del aceite, los compuestos de hidrólisis son inocuos por ser similares a los originados por la acción de la lipasa pancreática, una vez ingerido el alimento graso.
En relación a las restantes determinaciones analíticas aplicadas a los aceites de fritura, la tabla III resume los resultados obtenidos. En primer lugar, puede observarse que los valores de la acidez libre se encuentran, en general, por debajo de $1 \%$, indicativo de un bajo nivel de hidrólisis. Sin embargo, en las tres muestras -6, 9 y 15- que contenían cantidades elevadas de compuestos hidrólisis, los valores de acidez son mucho más elevados, lo que hace prever una buena correlación entre el índice de acidez y las cantidades de ácidos grasos y diglicéridos.

La evaluación de dienos y trienos conjugados tiene también una clara significación en el análisis de aceites y grasas de fritura. La formación de dienos conjugados de los ácidos grasos poliinsaturados, previa a la formación de los compuestos primarios de oxidación -hidroperóxidos- originó un incremento apreciable de la absorción a $232 \mathrm{~nm}$ de los aceites, a excepción de las muestras cuyo contenido en compuestos polares no experimentó un incremento significativo en relación a los aceites iniciales (muestras 4 y 11). A partir de estos resultados puede predecirse una buena relación entre este índice y la cantidad de compuestos de oxidación en las muestras. Los cambios, sin embargo, no fueron apreciables cuando se determinaron los contenidos en trienos conjugados en las muestras. El bajo incremento en la absorción a $270 \mathrm{~nm}$ en los aceites, característico de la formación de trienos conjugados, indicaría la poca importancia cuantitativa de la ruta de 
formación de compuestos volátiles en el proceso de fritura ya que los trienos conjugados más característicos que se encontrarían en los aceites serían los restos acilo diinsaturados que portan un grupo aldehído como consecuencia de la escisión del radical alcoxilo (Frankel, 1985; Kamal-Eldin et al., 1977).

Respecto al índice de peróxidos, es bien conocido que no constituye una buena medida de la alteración de las grasas 0 aceites de fritura ya que, aunque se originan en cantidades elevadas, a la temperatura del proceso de fritura su velocidad de descomposición es superior a la velocidad de formación (Stevenson, 1984). En consecuencia, los valores obtenidos en las muestras son más indicativos de la oxidación que tuvo lugar a baja temperatura una vez concluido el calentamiento. Su aplicación en este estudio tiene como objetivo comprobar si la determinación es de utilidad al menos cuando los aceites tienen similar grado de insaturación. Como puede comprobarse, los valores obtenidos son en todos los casos bajos, ya que las muestras se mantienen congeladas hasta el momento de los análisis, y no parecen tener relación con la alteración de las muestras.

La determinación de ácidos oxidados insolubles en éter de petróleo proporcionó resultados sorprendentes, particularmente en las muestras iniciales. El método es de interés por ser la base de la primera recomendación establecida en Alemania para desechar las grasas de fritura para consumo humano (Guhr y Waibel, 1979). Como se ha comentado, el valor fijado, entre 0,7 y $1 \%$, se encontró ya en algunas muestras iniciales cuyos restantes parámetros indicaban, sin embargo, características de calidad muy aceptables (muestras 1, 3, 7, 11 y 13 en tabla I). No obstante, los valores en los aceites de fritura fueron, en general, superiores a los encontrados en los aceites iniciales. Dado que la determinación es lenta, tediosa y difícil de interpretar, su sustitución por la evaluación de compuestos polares para decidir el momento en que la grasa debe desecharse supone una simplificación y mejora tan indudables, que la determinación deja de tener justificación en el análisis de las grasas de fritura.

El incremento de la viscosidad en los aceites y grasas de fritura está relacionado con la formación de compuestos de polimerización, los más característicos entre los diferentes grupos originados en el proceso de fritura. Como puede observarse en la tabla III, los resultados obtenidos en las muestras de aceites de fritura fueron en todos los casos superiores a los valores iniciales (tabla I), siendo el aumento tanto mayor cuanto más elevada es la cantidad de compuestos de polimerización.

Finalmente, en la última columna de la tabla III se recogen los resultados obtenidos utilizando la prueba colorimétrica comercializada con el nombre de Oxifrit, basada en el cambio de color de un indicador de oxidación-reducción cuyo viraje depende de la cantidad de compuestos de oxidación (Mayer, 1979). La obtención de un color azul o azul-verde en las condiciones de ensayo, indica que la grasa tiene aún calidad suficiente para seguir en uso (resultado negativo), mientras que la aparición de un color verde significa que debe ser desechada (resultado positivo), lo que de acuerdo con las normas y recomendaciones existentes debería ocurrir para valores del $25 \%$ de compuestos polares. Como puede observarse en la tabla III, los resultados obtenidos fueron excelentes ya que todas las muestras con porcentajes de compuestos polares por debajo del $25 \%$ dieron resultado negativo y sólo la muestra de mayor alteración con $25,4 \%$ de compuestos polares dio positiva.

En la tabla IV se incluyen los coeficientes de regresión lineal obtenidos para todas las determinaciones aplicadas en este estudio, que permiten deducir las medidas de mayor interés para evaluar de forma indirecta la degradación de la grasa de fritura y en la tabla $V$ se dan las rectas de regresión de las combinaciones para las que se obtuvo una correlación significativa y sus respectivos niveles de significación. Sólo se ha omitido la prueba colorimétrica ya que posee una escala discontinua y no tiene como objetivo evaluar la calidad de los aceites de fritura a partir del resultado sino decidir si la grasa debe o no ser desechada.

Dada la preponderancia de la determinación de compuestos polares en la evaluación de la calidad de los aceites y grasas de fritura, lo ideal sería encontrar una medida simple para su sustitución. El análisis de los coeficientes indica que se obtienen correlaciones significativas con los grupos de compuestos relacionados con la alteración termooxidativa -triglicéridos poliméricos, triglicéridos monómeros oxidados y ácidos oxidados- como se ha descrito previamente (Arroyo et al., 1992), aunque tienen mayor interés práctico las correlaciones de los compuestos polares con la viscosidad y el contenido en dienos conjugados. Los resultados demuestran, además, de forma indirecta, que la contribución de los compuestos de hidrólisis al total de compuestos de alteración es aleatoria.

Por otra parte, puede observarse que los valores de los índices de peróxidos y trienos conjugados no mostraron correlaciones significativas con ninguna de las restantes determinaciones. Teniendo en cuenta la homogeneidad de los aceites y del alimento utilizado, los resultados indicarían la falta de validez de las citadas determinaciones para evaluar la calidad de los aceites y grasas de fritura.

Entre los índices utilizados, sólo la medida de la viscosidad y la cantidad de dienos conjugados mostraron correlaciones consistentes con los compuestos característicos de la alteración. La elevada correlación entre ellos $(0,88)$ indica que sería redundante la aplicación de ambos para la evaluación indirecta de la alteración ya que el incremento de la formación de 
Tabla IV

Coeficientes de correlación entre determinaciones analíticas

\begin{tabular}{|c|c|c|c|c|c|c|c|c|c|c|}
\hline & $\begin{array}{c}\text { Compuestos } \\
\text { Polares }\end{array}$ & Polímeros & $\begin{array}{c}\text { Monómeros } \\
\text { oxidados }\end{array}$ & $\begin{array}{l}\text { Compuestos } \\
\text { de hidrólisis }\end{array}$ & $\begin{array}{l}\text { Acidez } \\
\text { Libre }\end{array}$ & $\begin{array}{l}\text { Indice } \\
\text { Peróxidos }\end{array}$ & Dienos & Trienos & $\begin{array}{l}\text { Acidos } \\
\text { Oxidados }\end{array}$ & Viscosidad \\
\hline \multicolumn{11}{|l|}{ Compuestos } \\
\hline Polares & 1,00 & $0,73^{\star \star}$ & $0,57^{\star}$ & 0,43 & 0,46 & 0,30 & $0,64^{\star \star}$ & 0,45 & $0,72^{\star \star}$ & $0,63^{*}$ \\
\hline Polímeros & & 1,00 & $0,90^{\star \star *}$ & 0,29 & 0,23 & 0,30 & $0,80^{\star \star *}$ & 0,06 & $0,83^{\star \star \star}$ & $0,81^{\star \star \star}$ \\
\hline \multicolumn{11}{|l|}{ Monómeros } \\
\hline oxidados & & & 1,00 & 0,47 & 0,38 & 0,41 & $0,74^{* *}$ & 0,04 & $0,68^{\star *}$ & $0,84^{\star \star \star}$ \\
\hline \multicolumn{11}{|l|}{ Compuestos } \\
\hline de Hidrólisis & & & & 1,00 & $0,95^{* * *}$ & 0,04 & 0,16 & 0,51 & 0,05 & 0,23 \\
\hline Acidez Libre & & & & & 1,00 & 0,07 & 0,09 & 0,44 & 0,02 & 0,22 \\
\hline \multicolumn{11}{|l|}{ Indice de } \\
\hline Peróxidos & & & & & & 1,00 & 0,39 & 0,20 & 0,28 & 0,35 \\
\hline \multicolumn{11}{|l|}{ Dienos } \\
\hline Conjugados & & & & & & & 1,00 & 0,15 & $0,55^{\star}$ & $0,88^{\star \star *}$ \\
\hline \multicolumn{11}{|l|}{ Trienos } \\
\hline Conjugados & & & & & & & & 1,00 & 0,07 & 0,01 \\
\hline \multicolumn{11}{|l|}{ Acidos } \\
\hline Oxidados & & & & & & & & & 1,00 & $0,55^{\star}$ \\
\hline Viscosidad & & & & & & & & & & 1,00 \\
\hline
\end{tabular}

${ }^{\star} p<0,05 ;{ }^{\star \star} p<0,01 ;{ }^{\star \star *} p<0,001$

Tabla V

Relaciones entre Métodos Analíticos

\begin{tabular}{|c|c|c|c|}
\hline $\mathbf{x}$ & $\mathbf{Y}$ & $\begin{array}{l}\text { Ecuación de la } \\
\text { recta de regresión }\end{array}$ & $\begin{array}{c}\text { Significación } \\
\text { (F) }\end{array}$ \\
\hline Compuestos Polares & Polímeros & $y=0,45 x-0,4$ & $1,9 \times 10^{-3}$ \\
\hline Compuestos Polares & Monómeros Oxidados & $y=0,21 x+1,0$ & $2,7 \times 10^{-2}$ \\
\hline Compuestos Polares & Dienos Conjugados & $y=0,06 x+0,46$ & $9,7 \times 10^{-3}$ \\
\hline Compuestos Polares & Ácidos Oxidados & $y=0,06 x+0,15$ & $2,2 \times 10^{-3}$ \\
\hline Compuestos Polares & Viscosidad & $y=0,51 x+29,5$ & $1,1 \times 10^{-2}$ \\
\hline Polímeros & Monómeros Oxidados & $y=0,54 x+0,8$ & $4,0 \times 10^{-6}$ \\
\hline Polímeros & Dienos Conjugados & $y=0,13 x+0,60$ & $3,7 \times 10^{-4}$ \\
\hline Polímeros & Viscosidad & $y=1,05 x+30,4$ & $2,3 \times 10^{-4}$ \\
\hline Polímeros & Ácidos Oxidados & $y=0,11 x+0,34$ & $1,3 \times 10^{-4}$ \\
\hline Monómeros Oxidados & Dienos Conjugados & $y=0,20 x+0,56$ & $1,6 \times 10^{-3}$ \\
\hline Monómeros Oxidados & Ácidos Oxidados & $y=0,15 x+0,39$ & $5,2 \times 10^{-3}$ \\
\hline Monómeros Oxidados & Viscosidad & $y=1,82 x+29,4$ & $9,2 \times 10^{-5}$ \\
\hline Compuestos de Hidrólisis & Acidez Libre & $y=0,26 x-0,11$ & $4,1 \times 10^{-8}$ \\
\hline Dienos Conjugados & Ácidos Oxidados & $y=0,46 x+0,37$ & $3,5 \times 10^{-2}$ \\
\hline Dienos Conjugados & Viscosidad & $y=7,11 x+26,9$ & $1,5 \times 10^{-5}$ \\
\hline Ácidos Oxidados & Viscosidad & $y=5,28 x+31,3$ & $3,4 \times 10^{-2}$ \\
\hline
\end{tabular}


polímeros -más relacionado con la viscosidad-, es también paralelo a la formación de dienos conjugados.

Finalmente, es también interesante resaltar que la correlación más elevada se encuentra entre la acidez libre y los compuestos de hidrólisis, lo que permitiría predecir a partir de un índice simple la cantidad de diglicéridos y ácidos grasos presentes en la muestra. Tal predicción sería de especial interés en las muestras de elevada acidez.

En resumen, de los resultados globales obtenidos se deducirían las siguientes conclusiones:

- La fritura de un único producto y la utilización de aceites de similar grado de insaturación no permite predecir la evolución de los principales grupos de compuestos de alteración originados durante el proceso. Sería de particular interés definir la(s) variable(s) que dan lugar a una hidrólisis importante en algunos establecimientos.

- A partir de los valores de acidez y dienos conjugados (o viscosidad) sería posible predecir la degradación del aceite de fritura cuando se utilizan aceites de similar grado de insaturación.

- En este tipo de establecimiento, la prueba colorimétrica Oxifrit dio excelentes resultados. No obstante, dado el bajo contenido de compuestos polares de las muestras sería conveniente ampliar el número de resultados con aceites de elevado contenido en compuestos polares.

\section{AGRADECIMIENTOS}

Este trabajo fue financiado con fondos del proyecto FONDECYT 1950472. Se agradece la colaboración de la Srta. Aleida Kulikoff, Srta. Verónica Carvajal y Srta. Cynthia Vilches, por la realización de algunas determinaciones analíticas del presente trabajo.

\section{BIBLIOGRAFÍA}

AOCS (1993). - «Official methods and recommended practices of the American Oil Chemists Society", 3th edition.- AOCS, Champaign. Illinois.

Al-Kahtani, H. A. (1991). - «Survey of quality of used oils from restaurants".- J. Am. Oil Chem. Soc. 68, 857-862.

Arroyo, R., Cuesta, C., Sánchez-Montero, J. M. and SánchezMuniz, F. J. (1995). - «High performance size exclusion chromatography of palm olein used for frying".- Fat Sci. Technol. 97, 292-296.

Arroyo, R., Cuesta, C., Garrido-Polonio, C., López-Varela, S. and Sánchez-Muniz, F. J. (1992).- «High-performance size-exclusion chromatographic studies on polar components formed in sunflower oil used for frying".- J. Am. Oil Chem. Soc. 69, 557-563.

Croon, L. B., Rogstad, A., Leth, T. and Kiutamo, T. (1986). - «A comparative study of analytical methods for quality evaluation of frying fat".- Fette Seifen Anstrichm. 88, 87-91.

Cuesta, C., Sánchez-Muniz, F. J., Garrido-Polonio, C., LópezVarela, S. and Arroyo, R. (1993). - «Thermoxidative and hydrolytic changes in sunflower oil used in fryings with a fast turnover of fresh oil».- J. Am. Oil Chem. Soc. 70, 1069-1073.

Dobarganes, M. C. y Márquez Ruiz, G. (1995). -«Calidad de las grasas de fritura en el sector de restauración de Andalucía».- Grasas y Aceites 46, 115-120.

Dobarganes, M. C., Pérez-Camino, M. C. y Márquez Ruiz, G. (1989). - «Determinación de compuestos polares en aceites y grasas de fritura".- Grasas y Aceites 40, 35-38.

Dobarganes, M. C., Pérez-Camino, M. C. and Márquez Ruiz, G. (1988). - «High performance size exclusion chromatography of polar compounds in heated and nonheated fats".- Fat Sci. Technol. 90, 308-311.

Dobarganes, M. C., Márquez Ruiz, G. and Pérez-Camino, M. C. (1993). - «Thermal stability and frying performance of genetically modified sunflower seed (Helianthus annuus L.) oils".- J. Agric. Food Chem. 41, 678-681.

Firestone, D. (1996). - «Regulation of frying fat and oil» in "Deep Frying: Chemistry, nutrition and practical applications", pp. 323-334.- E. G. Perkins and M. D. Erickson (Ed.). - American Oil Chemists' Society, Champaign, Illinois.

Frankel, E. N. (1985). - "Chemistry of autoxidation: mechanism, products and flavor significance» in «Flavor chemistry of fats and oils", pp. 1-37.- D. M. Min and T. H. Smouse (Ed.).- American Oil Chemists' Society, Champaign, Illinois.

Gertz, Ch. (1986). - «Chromatographische Methoden bei der Untersuchung von Fritierfetten».- Fette Seifen Anstrichm. 88, 475-480.

Guhr, G. and Waibel, J. (1979). - «Chromatographische Methoden zur Bestimmung der Verdorbenheit von Fritierfetten».- Fette Seifen Anstrichm. 81, 511-519.

IUPAC (1987). - «Standard methods for the analysis of oils fat and derivatives».- 7th edition, Pergamon Press, Oxford.

Kamal-Eldin, A., Márquez Ruiz, G., Dobarganes, M. C. and Appelqvist, L. A. (1997). - «Characterization of aldehydic acids in used and unused frying oils and prefried french fries".- J. Chromatog. In press.

Meyer, Von H. (1979). -Eine neue und einfache Schnellmethode zur erfassung des oxidativen Zersetzungsgrades thermisch belasteter Fette».- Fette Seifen Anstrichm. 81, 524-525.

Orthoefer, F. T. and Cooper, D. S. (1996). - «Evaluation of used frying oil» in «Deep Frying: Chemistry, nutrition and practical applications», pp. 285-296.- E. G. Perkins and M. D. Erickson (Ed.).- American Oil Chemists'Society, Champaign, Illinois.

Pérez-Camino, M. C., Márquez Ruiz, G., Salgado Raposo, A. y Dobarganes, M. C. (1988). - «Alteración de grasas usadas en fritura III. Correlación entre índices analíticos y métodos de evaluación directa de compuestos de degradación».- Grasas y Aceites 39, 72-76.

Pérez-Camino, M. C., Márquez Ruiz, G., Ruiz Méndez, M. V. and Dobarganes, M. C. (1991). - «ipid changes during frying of frozen prefried foods".- J. Food Sci. 56, 16441650 .

Sebedio, J. L., Grandgirard, A., Septier, Ch. and Prevost, J. (1987). - «Etat d'altération de quelques huiles de friture prélevées en restauration».- Rev. Fr. Corps Gras 34, 15-18.

Sebedio, J. L., Bonpunt, A., Grandgirard, A. and Prevost, J. (1990). -Deep fat frying of frozen prefried french fries: Influence of the amount linolenic acid in the frying medium".- J. Agric. Food Chem. 38, 1862-1867.

Smith, L. M., Clifford, A. J., Hamblin, C. L. and Creveling, R. K. (1986). - "Changes in physical and chemical properties of shortening used for commercial deep-fat frying".- J. Am. Oil Chem. Soc. 63, 1017-1023. 
Steveson, S. G., Vaisey-Genser, M. and Eskin, N.A.M. (1984). - Quality control in the use of deep frying oils».- J. Am. Oil Chem. Soc. 61, 1102-1108.

Suys, L. (1991). - Contrôle de la qualité des graisses et huiles de friture: correlation entre teneur en composés polaires et viscosité".- Rev. Fr. Corps Gras 38, 219-224.

White, P. J. (1991). - «Methods for measuring changes in deep-fat frying oils".- Food Technol. 75-80.

Wolff, J. P., Mordret, F. X. and Dieffenbacher, A. (1991). - «Determination of polymerized triglycerides in oils and fats by high performance liquid chromatography".- Pure \& Appl. Chem. 63, 1163-1171.

Recibido: Mayo 1997

Aceptado: Julio 1997 